Journal of Engineering and Applied Sciences 15 (5): 1237-1243, 2020

ISSN: 1816-949X

(C) Medwell Journals, 2020

\title{
Development of an Algorithm to Enhance Routing Performance in the Internet of Things
}

\author{
Rahaf AL-Shaar, Yasser Jaamour and Khaldoun Khorzom \\ Department of Communications Engineering, \\ Higher Institute for Applied Sciences and Technology, Damascus, Syria
}

\begin{abstract}
The need for Internet of Thing 'IoT' applications is increasing day after day and now these applications are getting used in many domains and lots of systems. With this vast increasing, new problems arise with networks. Applying IoT in wide networks requires large number of sensor nodes in the network which will increase the data traffic in this network and subsequently losing more energy. Many solutions and protocols have been proposed to fix this problem and to adapt with these networks. The most important protocol was RPL but it has many weaknesses: like inefficiency with load balancing. In this study, we propose a solution to alleviate the problem of losing energy and to improve the performance of RPL routing protocol. We propose an algorithm which uses an efficient strategy based on 4 metrics to choose the preferred parent (Node rank, ETX metric, residual energy and number of transmitted packets per node). The algorithm had been implemented using Cooja simulator. In addition, the proposed algorithm had been evaluated considering average power consumption and Packet Delivery Ratio (PDR) of different-densities networks. Plus, a precise comparison between the proposed algorithm and ECRM algorithm had been accomplished. Simulation results show that our proposed algorithm outperforms ECRM algorithm in terms of improving the process of energy consumption and packet transmitting rate.
\end{abstract}

Key words: IoT, load balancing, RPL, power consumption, packet, ECRM

\section{INTRODUCTION}

Internet of Things (IoT) is a recent communication paradigm that envisions a near future in which the objects of everyday life will be equipped with micro controllers, transceivers for digital communication and suitable protocol stacks that will make them able to communicate with one another and with the users, becoming an integral part of the internet (Zanella et al., 2014).

Most of IoT applications use Low power and Lossy Network(LLN) networks which have sensor nodes with constrained resources these networks are limited in term of memory, processing and energy consumption (Sebastian and Sivagurunathan, 2018). Therefore, routing protocols should be more suitable to fit with that and fulfil all application requirements that related to energy efficiency to prolong network lifetime in addition to rise the reliability and minimize the latency.

In 2012, IETF considered IPv6 Routing Protocol for Low power and lossy network (RPL) the standard routing protocol of LLN networks (Winter et al., 2012). RPL protocol chooses paths depending on routing metrics and objective function but the problem here is that there are many weaknesses like load imbalance (Qasem et al., 2018) which causes additional consumption of nodes energy. To solve this problem, an algorithm has been proposed to improve load balance through reducing energy consumption which leads to prolonging network lifetime.

The proposed algorithm has been created to fit IoT applications which require low-energy consumption and long lifetime network and to adapt to networks with large number of sensor nodes, like smart cities where there are large number of devices with very limited potentials in battery, processor and memory in addition to applications that require data collecting like environmental monitoring. The rest of this study would be divided as follows: an overview of RPL protocol will be over viewed in the 2 nd part. In the 3rd part we will present some important related works available in the literature; our proposed algorithm will be presented in the 4th part in the 5 th part we will evaluate the performance of our algorithm, finally, a future prospects of this study will be proposed.

\section{Overview of RPL protocol}

Control messages: RPL protocol is considered as a standard routing protocol of LLN networks, it creates the network topology by a graph called DAG which consists one or more Destination-Oriented Direct Acyclic Graphs (DODAGs). RPL uses 3 types of controlling messages to build DODAG which are (Conta et al., 2006; Aljarrah et al., 2016).

Corresponding Author: Rahaf AL-Shaar, Department of Communications Engineering, Higher Institute for Applied Sciences and Technology, Damascus, Syria 
DODAG Information Object (DIO): DIO message contains network parameters that allows node to find an RPL instance, maintain the DODAG construction, find its own setting coefficients and choose the list of parents in the network.

DODAG Information Solicitation (DIS): DIS is usually used by any node to explicitly solicit DIO messages directly from neighbouring nodes, it is also used to add nodes to DODAG tree if it was impossible to receive DIO message after a predefined time interval.

DAODestination Advertisement Object: DAO message carries destination information upward along DODAG tree which means providing data of the ascending path by registering every node has been visited through the specific ascending path. Each node except DODAG root sends DAO message to transmit prefixes and children routing tables to parents.

Constructing DODAG tree: First of all, the root node starts building DODAG by broadcasting DIO message to neighbouring nodes which contains a lot of information like node rank (allow nodes to locate themselves in DODAG and avoid routing loops), operation mode, objective functions and metrics.

Each node receives a DIO message should process the message and take decision if it would join DODAG or not according to $\mathrm{OF}$ being in use. If the node joins DODAG, it will have an ascending path upward to the root then it determines its rank and updates its own neighbouring nodes table and chooses the preferred parent which would be used to forward messages to DODAGE root. The preferred parent is usually the neighbouring node that has the minimum rank according to the OF. Each node receives DIO message must process it and repeat the whole process until it is delivered to all nodes in the network (Sobral et al., 2019).

RPL allows new nodes to be joined. Every new node use DIS message to request DIO message from another node which is already existed in DODAG.

Objective Function (OF): DODAG building process depends on the objective functions that network considers, OF combine many different metrics to calculate the rank and make the protocol use this rank to create optimal paths. Researchers put two standards to $\mathrm{OF}$ in RPL which are:

Objective Function Zero (OF0) which relates to hop count as a routing metric to specify the preferred parent among all the candidate neighbouring nodes (Thubert, 2012).

Minimum Rank with Hysteresis Objective Function (MRHOF) which select the best path based on ETX (Expected Transmission Count) which is the maximum number of resending processes that are needed to receive packets correctly in the destination.
Literature view: Load-balancing is still an open issue in RPL, especially with the fact that there are more than one possibility to choose the parent which means, either that the parent node is under very heavy $t$ raffic or there is no traffic at all. Better solutions for such problems are still envisaged in order to improve routing and prolonging network lifetime and managing the constrained resources efficiently.

Researchers in Sharkawy et al. (2014) have created Context Aware Objective Function (CAOF) that allows RPL to consider constrained resources of sensor nodes and its time changes, like battery level.

In Al-Kashoash et al. (2016) the researchers used the Buffer Occupancy (BO) with ETX as a complex metric to balance traffic and minimize the number of lost packets. In Taghizadeh et al. (2018) the researchers suggested solutions to fix the problem of missing packets and energy over consumption when the traffic is heavy they suggested a new routing metric called (Contest Aware Routing metric (CARF)) this metric considers using buffers and parents residual energy and it helps network with having more balanced energy and more paths to balance the load.

\section{MATERIALS AND METHODS}

The concerned algorithm and our proposed enhancement: RPL protocol has been designed to cover the increasing requirement for resource constrained devices but as we have already mentioned, it has balancing-load problem which reduces the network reliability. This problem leads to a huge difference in data traffic which in turn leads in the heavily loaded nodes to consume additional energy faster than other regular nodes. That would cause energy over consumption and force part of the network to be disconnected.

To improve the network performance and solve this problem, Energy-and Congestion-aware Routing Metric (ECRM) algorithm has been proposed (Ullah etal., 2017). This algorithm considers the residual energy of a node in a manner that the parent node with energy level under a certain threshold will not be selected in order to avoid disconnection in its children nodes. The algorithm also considers the queue utilization in the nodes which are elected to be the best parent to prevent any overflow in the queues which might leads to consecutive packet drop. We suggested an improvement of the algorithm using these metrics:

- Residual energy and consumed energy of each node In that case the connected part of the network would not be disconnected as its energy depletes

- ETX metric, in order to choose high quality links

- Number of packets sent to each node in order to avoid congestion and overload in the network

- Node ranks 


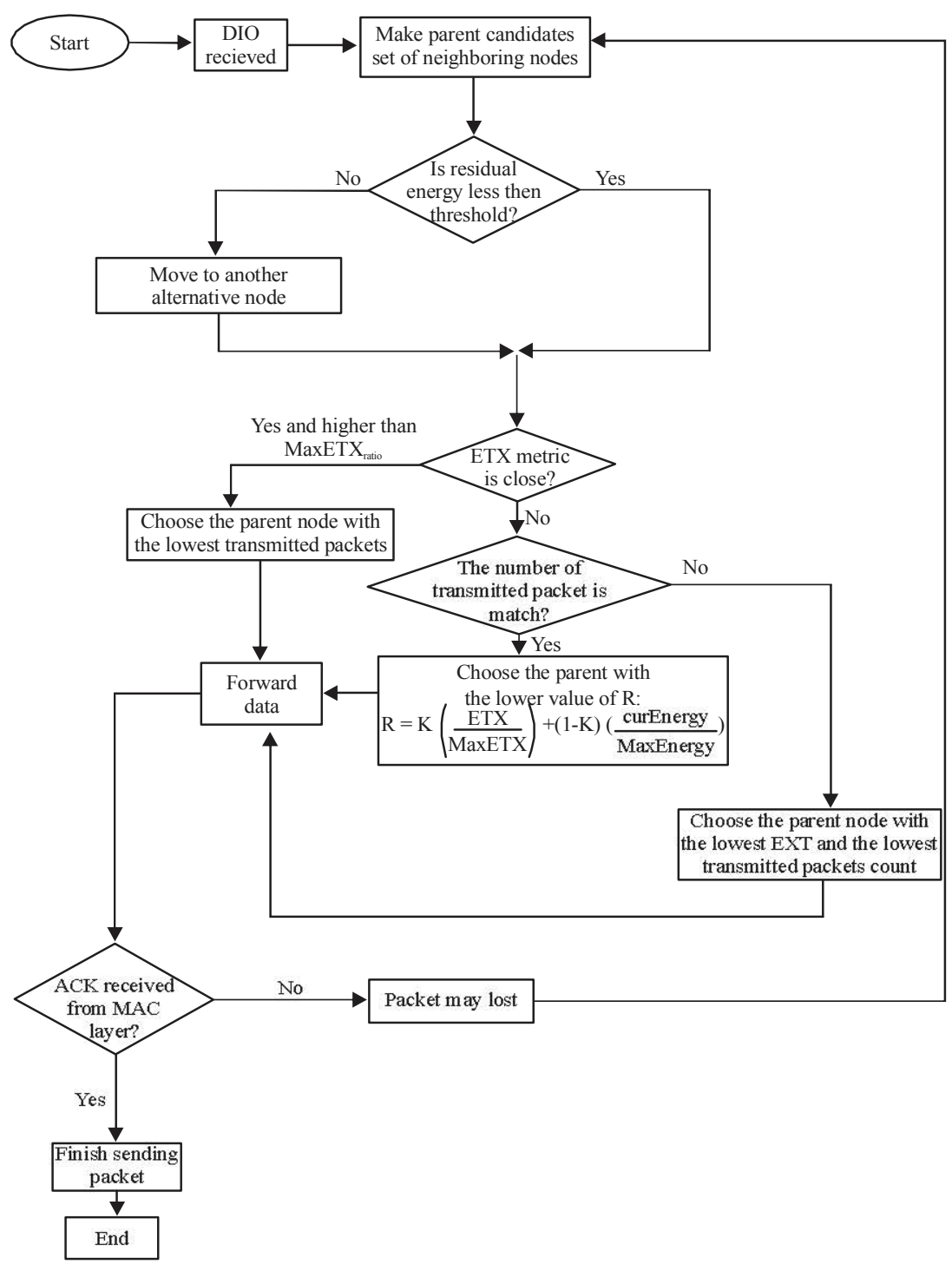

Fig. 1: Process of the proposed scheme

The steps of the algorithm are as follow: each node recognizes its neighbours by DIO messages which contain information like rank, ETX, energy status and transmitted packets count. Form a group of parent candidates.

Each node selects the parent node according to the lowest ETX value, the smallest number of transmitted packets and a certain threshold of the residual energy. The algorithm would be applied on every pair of parents as: first, we check if the residual energy of each parent node is over the threshold or we will move to another alternative node.

We check the ETX metric and the proportion of transmitted packets, if ETX was in the acceptable range and there is difference in the number of transmitted packets then we would choose the parent node with the lowest ETX and the lowest transmitted packets count.
If ETX of two nodes are similar or even close and higher than max then we choose the parent node with the lowest transmitted packets count. Considering node $n$ and $\mathrm{p}_{1}$ and $\mathrm{p}_{2}$ as candidates parents of $\mathrm{n}$, if ETX $\mathrm{p}_{2}$ is the ETX metric of $p_{1}$ and ETX $p_{2}$ is the ETX metric of $p_{2}$ then the ETX metric ratio is calculated as follows (Fig. 1):

$$
\mathrm{EXT}_{\text {ratio }}= \begin{cases}\frac{\mathrm{EXT}_{\mathrm{P} 2} * 100}{\mathrm{EXT}_{\mathrm{P} 1}} & \mathrm{EXT}_{\mathrm{P} 1}>\mathrm{EXT}_{\mathrm{P} 1} \\ \frac{\mathrm{EXT}_{\mathrm{P} 1} * 100}{\mathrm{EXT}_{\mathrm{P} 2}} & \text { otherwise }\end{cases}
$$

If the transmitted packets count of parents are similar, then we choose the parent with the lower value of $R$. Where $\mathrm{R}$ is: 


$$
\mathrm{R}=\mathrm{K}\left(\frac{\mathrm{ETX}}{\text { MaxETX }}\right)+(1-\mathrm{K})\left(\frac{\text { cur energy }}{\text { Max energy }}\right)
$$
below:

The $\mathrm{K}$ value is the adjustable weight value as shown

$$
\mathrm{K}=\frac{\text { currank }}{\text { MaxRank }}
$$

Where:

currank : Represents a current node rank value Max rank : A maximum rank value in the networks

The R metric has a good energy balance in the lower ranks and good links in higher ranks because the low-rank node transmits a lot of data to a lot of children nodes, so, these nodes should have a long lifetime. On the other hand, the high-rank nodes should have an acceptable value of ETX to have rate of packets delivering.

After selecting the best parent, the data would be routed towards it. If an acknowledge packet is received from MAC, packet will be considered as successfully received by the parent node.

If no acknowledge packet was received from $\mathrm{MAC}$, packet would be lost and they have to be sent again and the process should be restarting from step 2. Figure 1 shows the schema of the proposed algorithm (Table 1).

Network setup: Simulation process runs through a random topology consisted of server node works as a root in DODAG and group of client nodes, the region density where nodes work is ranging between 20 nodes for low density and 100 nodes for high density. To introduce lossiness into the wireless medium we used UDGM Model which defines lossiness considering the relative distances of nodes in the radio medium and by this model we are able to control the ratio of transmitting and receiving. We kept the Transmission ratio (TX) 100\% because we consider the lossiness at the receiver and we changed the Reception Ratio (RX) which refers to the rate of the successful packets reception at the receiver and it also refers to the lossiness in the wireless medium. In fact, while the distance between a node and the other node's transmitting range decreases, the probability of successful

\begin{tabular}{ll} 
Table 1: Simulation parameters & \\
\hline Parameters & Values \\
\hline Simulator & Cooja Contiki 3.0 \\
Radio medium & UDGM \\
TX ratio & $100 \%$ \\
TX range & $50 \mathrm{~m}$ \\
INT range & $60 \mathrm{~m}$ \\
RX ratio & $20,40,60,80,100 \%$ \\
Energy model & Energest \\
Number of nodes & $20,40,60,80,100$ \\
DIO min & 12 \\
DIO doubling & 8 \\
Simulation time $2 \mathrm{~h}$ & $7200 \mathrm{sec}$ \\
MAC & 802.15 .4 \\
RDC channel check rate & $16 \mathrm{~Hz}$ \\
\hline
\end{tabular}

packets receiving increases in this node. We put the transmitting range on $50 \mathrm{~m}$ and the interference range on $60 \mathrm{~m}$, we run the simulation for $7200 \mathrm{sec}$, the Table 1 describes different parameters in the different scenarios.

\section{RESULTS AND DISCUSSION}

Energy consumption evaluation with fixed number of nodes and variable values of RX: In these scenarios, we evaluated the average energy consumption of each node using RX (the rate of successfully received packets at the receiver), we chose a fixed number of nodes and then we varied $R X$ values as in Fig. 2-4.

When fixing nodes number and taking two different values of RX (relatively good and bad cases), we noticed that average energy consumption would be higher when the value of RX is lower because when we increase the rate of successful receiving, lossiness in the network decreases and as this rate decreases the lossiness rate increases. In addition, networks with high lossiness requires additional transmitting processes and subsequently they consume more energy.

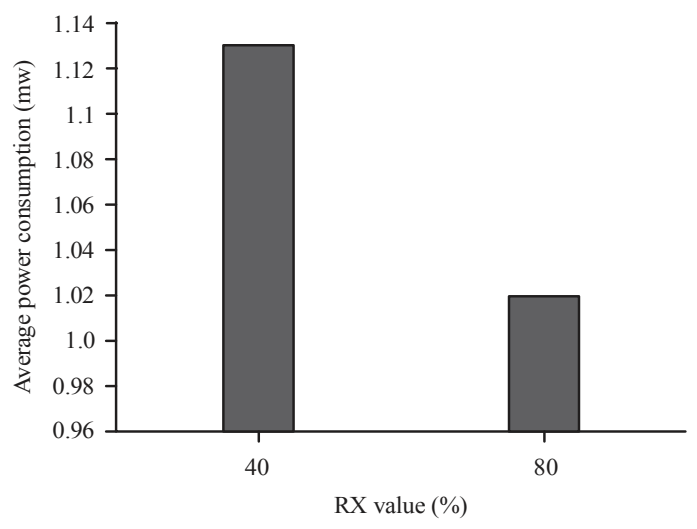

Fig. 2: Average power consumption as a function of the $\mathrm{RX}$ values with node density of 20

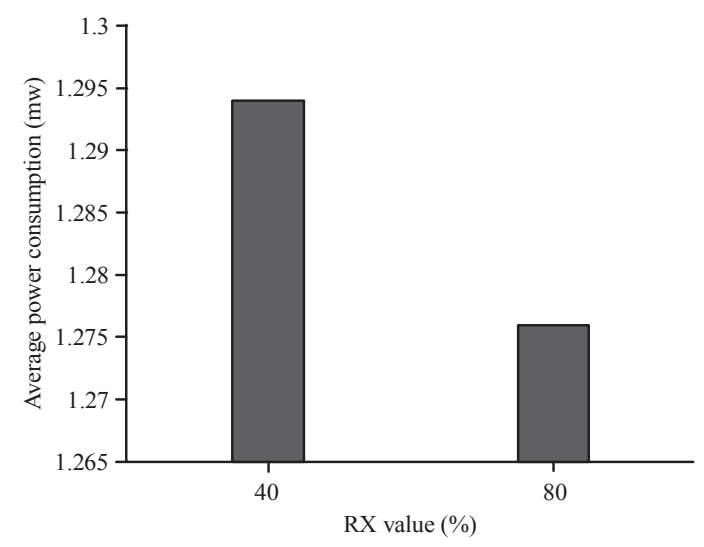

Fig. 3: Average power consumption as a function of the RX values with node density of 60 


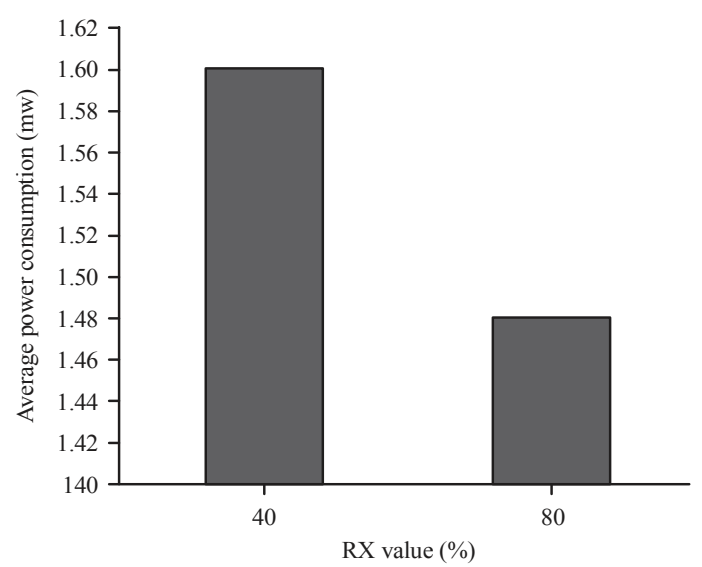

Fig. 4: Average power consumption as a function of the $\mathrm{RX}$ values with node density of 100

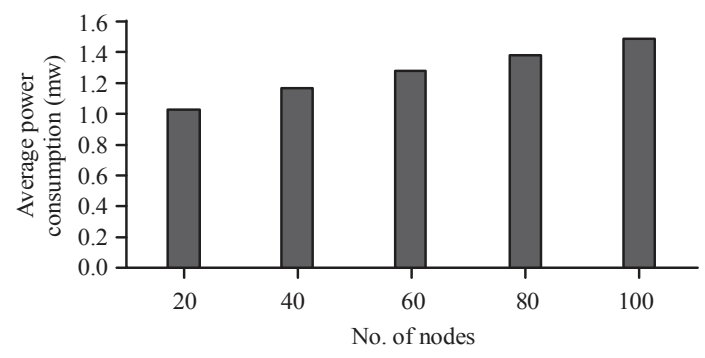

Fig. 5: Average power consumption as a function of the number of nodes when $\mathrm{Rx}=80 \%$



Fig. 6: Average power consumption as a function of the number of nodes when $\mathrm{Rx}=80$ and $40 \%$

Energy consumption evaluation with variable number of nodes and with fixed rates of RX: In these scenarios we evaluate the performance of the algorithm in terms of average energy consumption of variable-density network with fixed RX values.

Depending on the Fig. 5 and 6 , it is noticeable that when nodes count increases, energy consumption increases because the processes of transmitting and receiving packets increases. In another word, if traffic increases many nodes would run out of energy and its residual energy would be less than the threshold, so, the network is obliged to reselect the preferred parent and broadcast control messages again to build the network.

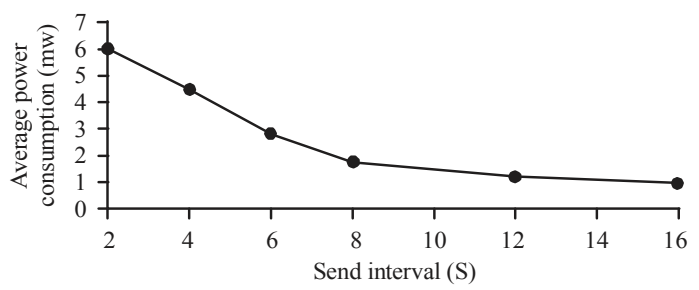

Fig. 7: Average power consumption as a function of send interval, $\mathrm{Rx}=80 \%$



Fig. 8: PDR as a function of the RX values with node density of 20

Energy consumption evaluation relating to send interval: In this scenario we evaluated the average energy consumption with time intervals between messages to make a relatively good rate of successful packet reception $\mathrm{RX}=80 \%$ as shown in Fig. 7. According to this figure we notice that the average energy consumption linearly decreases with send interval increasing. In another word, send interval is inversely proportional to energy consumption and as the rate of application messages increases, the network resources will be drained faster and the traffic will increase which causes more wireless collisions and energy over consumption.

Packet Delivery Ratio (PDR) evaluation with fixed number of nodes and variable values of $R X$ : We evaluated the packet delivery ratio with fixed number of nodes in all cases (low-density, high-density and medium-density) and we modified the ratio of successful packet reception at bad value and at relatively good value. According to Fig. 8-10, PDR decreases when the rate of successful packet reception is low comparing to the high rate of successful packet reception. This is because the energy consumption increases when RX decreases. The decrease of current parent's energy level requires searching for candidate nodes and reforming DODAG. This process causes packet loss and decrease in the PDR.

PDR evaluation with variable number of nodes and with fixed rates of RX: In these scenarios we evaluated the performance of the algorithm in terms of a packet delivery ratio of variable-density network with fixed RX values. 


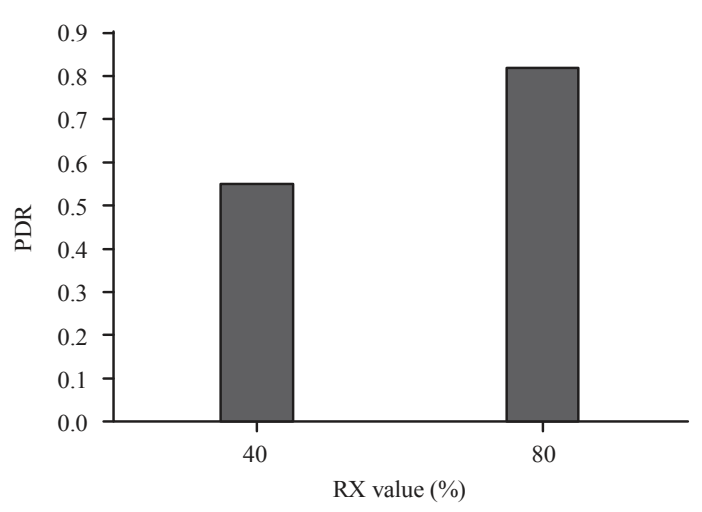

Fig. 9: PDR as a function of the RX values with node density of 60

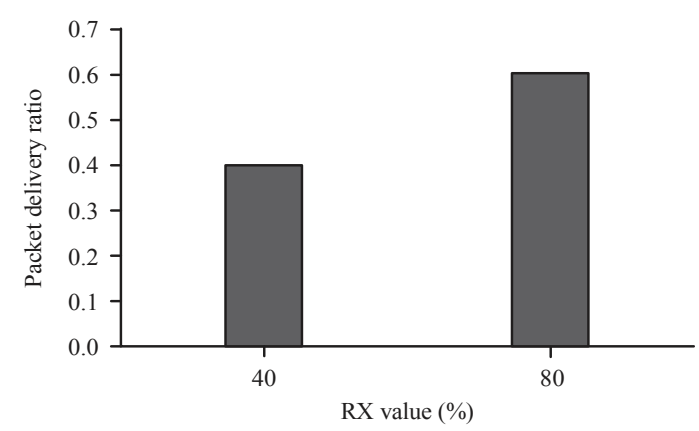

Fig. 10: PDR as a function of the RX values with node density of 100

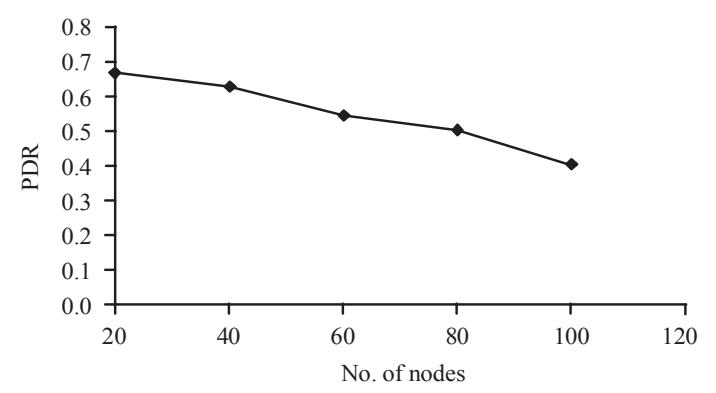

Fig. 11: $\mathrm{PDR}$ as a function of the number of nodes when $\mathrm{Rx}=40 \%$

According to Fig. 11 and 12, we notice that PDR remains almost close to each other when number of nodes increases. This is due to the improvement of energy consumption where nodes don't deplete the battery and subsequently, PDR stays relatively high.

Comparison of energy consumption between the proposed algorithm and ECRM algorithm: According to the comparison between the ECRM and our proposed algorithm, we notice that energy consumption in ECRM is higher as shown in Fig. 13 and 14. When node's buffer overflows a resending process is initiated which consumes

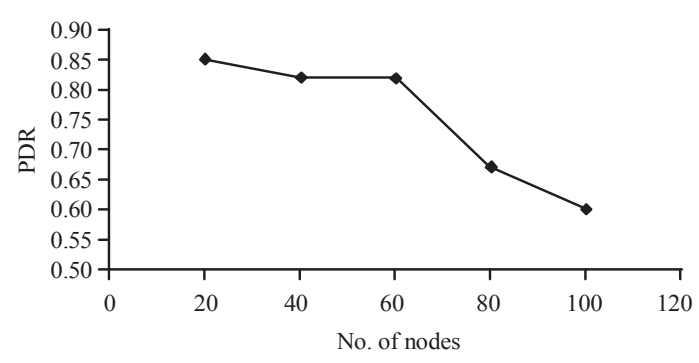

Fig. 12: PDR as a function of the number of nodes when $\mathrm{Rx}=80 \%$

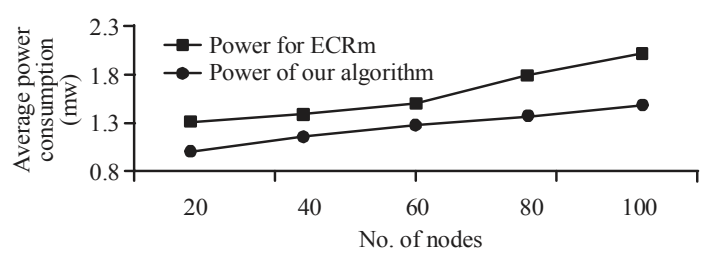

Fig. 13: Comparison of energy consumption between the proposed algorithm and ECRM algorithm in best channel conditions, power $\mathrm{Rx}=80 \%$

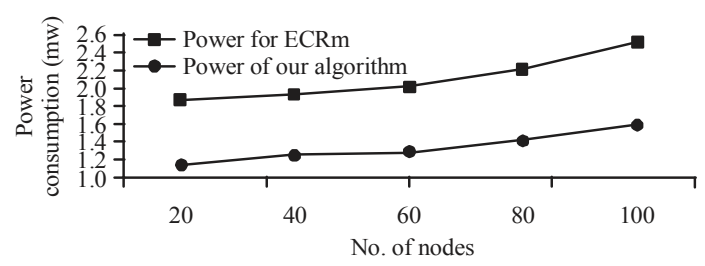

Fig. 14: Comparison of energy consumption between the proposed algorithm and ECRM algorithm in worst channel conditions, power $\mathrm{Rx}=40 \%$

more energy. In our proposed algorithm, energy consumption is better due to the fact that, parent who sends the least number of packet will be chosen as the preferred parent.

Comparison of PDR between our proposed algorithm and ECRM: In our proposed algorithm PDR is higher as shown in Fig. 15 and 16 comparing with ECRM. The enhanced performance of the proposed protocol is due to the improvement in energy consumption in the network through a good choice of the parent that sends less packets on the other hand in ECRM when number of nodes increases, the buffer capacity is exceeded and the processes of retransmission and reception increase too as a result the PDR decreases.

However, some complexity is added to our algorithm, so, a trade-off is needed between energy consumption and latency. Further investigations concerning CPU execution time are needed in order to fairly compare our approach with others. 


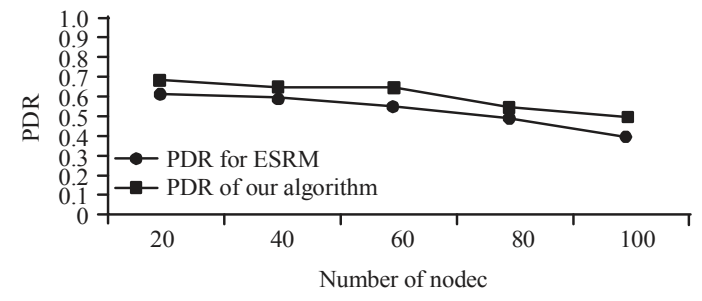

Fig. 15: Comparison of PDR between the proposed algorithm and ECRM algorithm in best channel conditions, $\mathrm{PDR}, \mathrm{RX}=80 \%$

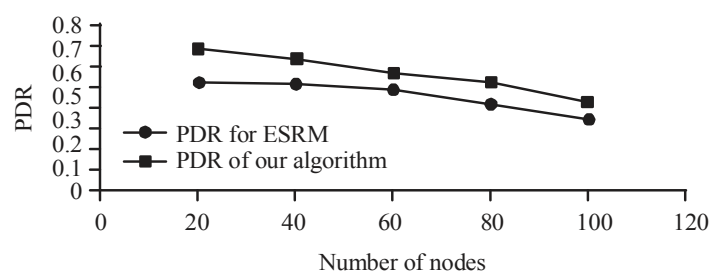

Fig. 16: Comparison of PDR between the proposed algorithm and ECRM algorithm in worst channel conditions, PDR, RX $=40 \%$

\section{CONCLUSION}

In this study, we proposed an algorithm for load-balancing in RPL through balancing the energy consumption, considering metrics like residual energy of nodes, transmitting packets count, ETX and node rank.

This algorithm shown an improvement in energy consumption all over the network, and in the PDR also. Our algorithm outperforms ECRM in terms of energy consumption and PDR.

Future work: Some additional future works still to be elaborated: evaluating the algorithm performance for different metrics in different scenarios.

Improving the performance of the proposed algorithm through modifying and improving many parameters like DIO minimum interval, DIO interval doublings and radio duty-cycling interval. These parameters are very important in controlling the general traffic, energy consumption and convergence time. Studying and evaluating the algorithm performance with mobile nodes in the network.

\section{REFERENCES}

Al-Kashoash, H.A., Y. Al-Nidawi and A.H. Kemp, 2016. Congestion-aware RPL for 6LOWPAN networks. Proceedings of the 2016 International Wireless Telecommunications Symposium (WTS'16), April 18-20, 2016, IEEE, London, UK., pp: 1-6.
Aljarrah, E., M.B. Yassein and S. Aljawarneh, 2016. Routing protocol of low-power and lossy network: Survey and open issues. Proceedings of the 2016 International Conference on Engineering \& MIS (ICEMIS'16), September 22-24, 2016, IEEE, Agadir, Morocco, pp: 1-6.

Conta, A., S. Deering and M. Gupta, 2006. Internet Control Message Protocol (ICMPV6) for the Internet Protocol Version 6 (IPV6) specification. http://tools.ietf.org/pdf/rfc4443.pdf.

Qasem, M., A. Al-Dubai, I. Romdhani and B. Ghaleb, 2018. Load balancing objective function in RPL draft-qasem-roll-rpl-load-balancing-01. IETF, Internet Engineering Task Force, Fremont, California, USA. https://www.napier.ac.uk/researchand-innovation/research-search/outputs/loadbalancing-objective-function-in-rpl-draft-qasem-rollrpl-load-balancing-01

Sebastian, A. and S. Sivagurunathan, 2018. Load balancing metric based routing protocol for low power and lossy networks (lbRPL). Int. J. Eng. Technol., 7: 39-44.

Sharkawy, B., A. Khattab and K.M. Elsayed, 2014. Fault-tolerant RPL through context awareness. Proceedings of the 2014 IEEE World Forum on Internet of Things (WF-IoT'14), March 6-8, 2014, IEEE, Seoul, South Korea, pp: 437-441.

Sobral, J.V., J.J. Rodrigues, R.A. Rabelo, J. Al-Muhtadi and V. Korotaev, 2019. Routing protocols for low power and lossy networks in internet of things applications. Sensors, Vol. 19, No. 9. $10.3390 / \mathrm{s} 19092144$

Taghizadeh, S., H. Bobarshad and H. Elbiaze, 2018. CLRPL: Context-aware and load balancing RPL for IoT networks under heavy and highly dynamic load. IEEE. Access, 6: 23277-23291.

Thubert, P., 2012. Objective function zero for the routing protocol for low-power and lossy networks. Internet Eng. Task Force (IETF), Vol. 1,

Ullah, R., Y. Faheem and B.S. Kim, 2017. Energy and congestion-aware routing metric for smart grid AMI networks in smart city. IEEE. Access, 5: 13799-13810.

Winter, T., P. Thubert, A. Brandt, T. Clausen and J. Hui et al., 2012. RPL: IPv6 routing protocol for low power and lossy networks. Internet Eng. Task Force (IETF), Vol. 1,

Zanella, A., N. Bui, A. Castellani, L. Vangelista and M. Zorzi, 2014. Internet of things for smart cities. IEEE Internet Things J., 1: 22-32. 